\title{
Resistencia antihelmíntica en nematodos gastrointestinales de ovinos tratados con ivermectina y fenbendazol
}

\author{
Anthelmintic resistance of gastrointestinal nematode in sheep \\ treated with ivermectin and fenbendazole.
}

\author{
A Toro ${ }^{\mathrm{a}}$, L Rubilar ${ }^{\mathrm{b} \dagger}$, C Palma ${ }^{\mathrm{a}}$, R Pérez ${ }^{\mathrm{a}}$ \\ aLaboratorio de Farmacología, Departamento de Ciencias Clínicas, Facultad de Ciencias Veterinarias, \\ Universidad de Concepción, Campus Chillán, Chillán, Chile. \\ bLaboratorio de Parasitología y Enfermedades Parasitarias, Departamento de Patología y Medicina Preventiva, \\ Facultad de Ciencias Veterinarias, Universidad de Concepción, Campus Chillán, Chillán, Chile.
}

\begin{abstract}
SUMMARY
The aim of the study was to evaluate the presence of anthelmintic resistance in gastrointestinal nematodes of infected sheep treated with ivermectin (IVM) and fenbendazole (FBZ). Thirty six Suffolk Down breed, young-female of eight months of age and $37.2 \pm 4.0 \mathrm{~kg}$ of body weight (bw) were distributed in three experimental groups of 12 animals each. Group I (Control) was non treated. Group II was treated with IVM at $0.2 \mathrm{mg}$ per $\mathrm{kg}$ bw subcutaneously. Group III was treated with $5 \mathrm{mg} \mathrm{FBZ/kg} \mathrm{bw} \mathrm{by} \mathrm{oral} \mathrm{route.} \mathrm{Faecal} \mathrm{samples} \mathrm{were} \mathrm{obtained} \mathrm{at} 0,7$ and 15 days after treatments and processed for faecal egg count test and then faecal egg count reduction test (FECRT) was performed. Group II treated with IVM showed a FECRT of 34 and $77 \%$ on days 7 and 15 post-treatment, respectively. The lower limit of $95 \%$ interval confidence (LL-IC ${ }_{95 \%}$ ) for this group was $38 \%$. No larval hatching was observed in egg cultures from samples of faeces of sheep treated with IVM. Group III showed an egg count reduction of 74\% with a LL-IC 95\% of 40\%, the genera with the highest frequency of resistance were Teladorsagia and Trichostrongylus. In conclusion, the gastrointestinal nematodes of the studied sheep are resistant to the anthelmintics IVM and FBZ.
\end{abstract}

Key words: resistance, anthelmintics, sheep, parasites.

\section{RESUMEN}

Se realizó un estudio con el objetivo de evaluar la presencia de resistencia en nematodos gastrointestinales de ovinos tratados con los antihelmínticos ivermectina (IVM) y fenbendazol (FBZ). Se utilizaron 36 borregas raza Suffolk Down de 8 meses de edad, con pesos promedios de 37,2 kg, las que fueron distribuidas en 3 grupos de 12 animales. Grupo I, sin tratamiento (control), Grupo II, tratado con 0,2 mg/kg de IVM vía subcutánea. Grupo III, tratado con $5 \mathrm{mg} / \mathrm{kg}$ de FBZ vía oral. Se obtuvieron muestras fecales los días 0,7 y 15 posteriores al tratamiento. Se determinó el porcentaje de reducción del recuento fecal de huevos de nematodos $(\mathrm{PRCH})$ y se le calculó el límite inferior del intervalo al $95 \%$ de confianza $\left(\mathrm{L}_{\text {inf }}-\mathrm{IC}_{95 \%}\right)$. Se realizaron coprocultivos para la determinación de los géneros parasitarios. Las borregas del Grupo II tratado con IVM, presentaron PRCH de $34 \%$ el día 7 y de $77 \%$ el día 15 . El $\mathrm{L}_{\mathrm{inf}}-\mathrm{IC}_{95 \%}$ para este grupo fue de $38 \%$. En el coprocultivo no se observó desarrollo de larvas. El Grupo III tratado con FBZ, presentó $\mathrm{PRCH}$ de 41 y $74 \%$ para los días 7 y 15 respectivamente, con un $\mathrm{L}_{\text {inf }}-\mathrm{IC}_{95 \%}$ de $40 \%$. En este grupo los géneros que presentaron el mayor porcentaje de eclosión de larvas fueron Teladorsagia y Trichostrongylus. Se concluye que en las borregas estudiadas los nematodos gastrointestinales presentan resistencia a IVM y FBZ.

Palabras clave: resistencia, antihelmínticos, ovinos, parásitos.

\section{INTRODUCCIÓN}

Las infecciones por nematodos gastrointestinales constituyen una limitante en la producción de rumiantes con efectos que varían desde pérdidas subclínicas de peso hasta la muerte de animales severamente parasitados. Estos nematodos son de localización cosmopolitas, están inevitablemente asociados a los animales en pasto-

Aceptado: 03.10.2013

In memoriam (QEPD).

* Av. Vicente Méndez 595 Chillán, Chile; rubperez@udec.cl reo y toda práctica de producción animal que contemple el aprovechamiento racional de forrajes requiere de su control ${ }^{1}$.

En toda explotación ganadera el uso de antihelmínticos representa una de las herramientas más eficaces para el control del parasitismo en los animales de producción. El uso adecuado y racional de estos fármacos permite que los animales expresen su potencial productivo y

Anziani OS, CA Fiel. 2004. Resistencia de los nematodes gastrointestinales a los antihelmínticos: un problema emergente y relevante para la producción bovina nacional [en línea]. INTA, Argentina; http://cnia.inta.gov.ar/helminto/pdf\%20Resistencia/Anziani.pdf 
evita las pérdidas económicas que derivan del retraso en el desarrollo, la disminución de la ganancia de peso, la menor producción y en ocasiones la muerte de los animales debido a la acción patógena de los parásitos. Con la intensificación de los sistemas productivos, su uso se ha incrementado considerablemente por lo que actualmente estos fármacos representan el mayor segmento del mercado mundial de productos farmacéuticos de uso en animales (Pérez 2011).

Debido a la elevada eficacia de los antihelmínticos disponibles en el mercado y a la complejidad de utilizar otras metodologías de control, los productores basan toda su estrategia de prevención en el uso indiscriminado y continuo del recurso químico (Cristel y Suarez 2006). Como resultado de este manejo y la aplicación de estrategias desacertadas (Van Wyk 2001) se ha favorecido el desarrollo de resistencia a los antiheminticos.

La resistencia a los antihelmínticos se presenta con más frecuencia en ovinos que en bovinos (Williams 1997), debido principalmente a diferencias genético-fisiológicas entre las dos especies. Los pequeños rumiantes tienen dificultad para regular su parasitismo gastrointestinal (Jackson y Coop 2000), requiriendo un mayor número de tratamientos para mantener el estado sanitario.

Un estudio para determinar resistencia antihelmíntica en cuatro países del MERCOSUR (Argentina, Brasil, Paraguay, Uruguay) demostró una alta prevalencia del problema en nemátodos del ovino a nivel regional y la presencia de un importante número de poblaciones de nemátodos resistentes a las ivermectinas, situación que en esos momentos era más sospechada que conocida con argumentos científicos (FAO 2003). En Chile la presencia de parásitos resistentes al febendazol (FBZ) ha sido descrita en bovinos (Sievers y Fuentealba 2003, Sievers y Alocilla 2007) y equinos (Von Witzendorff y col 2003). Sin embargo, no existen antecedentes que demuestren la presencia de especies parasitarias resistentes a los diferentes grupos de antihelmínticos disponibles para uso en ovinos. Se realizó el presente estudio con el objetivo de evaluar la resistencia antihelmíntica de nemátodos gastrointestinales de ovinos frente a los fármacos ivermectina y fenbendazol.

\section{MATERIAL Y MÉTODOS}

El estudio se realizó entre los meses de mayo y junio de 2011, en un rebaño de 500 ovinos perteneciente al fundo "Bureo Alto", ubicado en la precordillera de la provincia de Ñuble a $48 \mathrm{~km}$ de la ciudad de Chillán, Región del Bío Bío, Chile. Se seleccionaron al azar 36 hembras ovinas de 6 a 8 meses de edad, de raza Suffolk Down con un peso promedio de 37,2 \pm 4,0 kg y con infección natural a nematodos gastrointestinales. Los animales se mantuvieron juntos en pastoreo en pradera mixta de trébol con ballica durante el día. En la noche eran encerradas en corrales del predio para evitar la acción de depredadores.
De cada animal se obtuvieron muestras fecales directamente del recto una semana antes del tratamiento y a los 0, 7 y 15 días posteriores al tratamiento. A cada muestra se le realizo un exámen coproparasitario para determinar el número de huevos de nemátodos mediante la técnica de McMaster, procedimiento que fue realizado en el Laboratorio de Parasitología del Departamento de Patología y Medicina Preventiva de la Facultad de Ciencias Veterinarias de la Universidad de Concepción, Campus Chillán.

\section{GRUPOS EXPERIMENTALES}

Los animales experimentales fueron borregas con cargas parasitarias promedios igual o mayor a 300 huevos por gramo de materia fecal, hpg (rango 300 - $1900 \mathrm{hpg}$ ). Los animales fueron pesados con una balanza electrónica con capacidad de hasta $150 \mathrm{~kg}$ y precisión $\pm 0,1 \mathrm{~g}$ (Shangai SP-150). Luego se dividieron en tres grupos de 12 animales cada uno. Las borregas fueron distribuidas mediante un diseño de bloques al azar considerando las variables del recuento de huevos de nematodos y peso corporal a los siguientes grupos experimentales:

Grupo 1, Grupo control. Constituido por 12 ovinos sin tratamiento antihelmíntico, con el fin de mantener la condición de parasitismo durante el muestreo. Este grupo al finalizar la investigación, recibió el respectivo tratamiento antihelmíntico.

Grupo 2, (IVM,). Animales tratados con $0,2 \mathrm{mg} / \mathrm{kg}$ de IVM al 1\% (Ivomec ${ }^{\circledR}$, Merial), administrada por vía subcutánea.

Grupo 3, (FBZ). Animales tratados con $5 \mathrm{mg} / \mathrm{kg}$ de FBZ en solución al 10\% (Panacur ${ }^{\circledR}$, Intervet) por vía oral.

\section{METODOLOGÍA}

Se determinó el recuento fecal de huevos de nematodos mediante la técnica de McMaster descrita por Barriga (2002), antes del tratamiento y a los días 7 y 15 posteriores al tratamiento con los antiparasitarios respectivos. Los resultados se expresan en huevos por gramo de materia fecal (hpg). Para el cultivo de larvas de nematodos gastrointestinales, se formaron dos "pool" de seis muestras de heces de cada grupo de animales por cada tiempo de muestreo (T0, T7 y T15), con un total de 18 cultivos. El cultivo se efectuó mediante el procedimiento de Corticelli y Lai descrito por Niec (1968). La recuperación de las larvas infectantes se obtuvo utilizando el método de Baermman, descrito por Barriga (2002). Posteriormente las larvas se inmovilizaron con lugol, y se observaron al microscopio para apreciar las características morfológicas y permitir su diferenciación. La diferenciación de las larvas, se efectuó tomando en consideración las principales características morfológicas tales como: largo total de la larva, forma y largo del esófago, largo de la vaina larval de la cola (distancia entre el ano y la punta de la cola), número de células intestinales y forma de éstas (Niec 1968). 


\section{ANÁLISIS DE RESULTADOS}

La eficacia antihelmíntica se determinó a través del porcentaje de reducción del recuento de huevos de nematodos (PRCH), y se calculó mediante el programa RESO desarrollado por la división de Salud Animal de CSIRO (Agencia Nacional para la Ciencia de Australia). Además, al PCRH se le determinó el límite inferior del intervalo de confianza al $95 \%\left(\mathrm{~L}_{\text {inf }}-\mathrm{IC}_{95 \%}\right)$, con un valor alfa de 0,05 .

Para la determinación del porcentaje de reducción de huevos de nematodos se utilizo la fórmula de Coles y col (1992),

$$
\operatorname{PRCH}(\%)=100(1-\bar{x} \text { GT/ } \bar{x} \text { GC })
$$

Dónde,

XG.C: Media aritmética del conteo de huevos del grupo control

X G.T: Media aritmética del conteo de huevos del grupo tratado

Según las recomendaciones de la World Association for the Advancement of Veterinary Parasitology (WAAVP), existe resistencia al antihelmíntico administrado cuando el porcentaje de reducción del recuento de huevos es menor al 95\% $\mathrm{y} \mathrm{L}_{\text {inf }}-\mathrm{IC}_{95 \%}$ es menor a $90 \%$. Asimismo, si solamente aparece uno de los dos parámetros anteriormente nombrados, se considera sospechoso de resistencia (Coles y col 1992).

El análisis estadístico de los datos se realizó mediante el programa InfoStat. Se utilizó la prueba de Bartlett para establecer la homogeneidad de las varianzas. Los prome- dios de recuentos de huevos de nematodos se compararon mediante un análisis de varianza asociado a una prueba de comparaciones múltiples. Se consideró un valor de $\mathrm{P}<0,05$ para establecer diferencias significativas entre los grupos.

\section{RESULTADOS}

\section{RECUENTO DE HUEVOS DE NEMÁTODOS EN MATERIA FECAL EN ANIMALES TRATADOS CON IVM Y FBZ}

En el cuadro 1 se presentan los promedios de recuentos de huevos de nemátodos en materia fecal obtenidos previo al tratamiento antihelmíntico. Para los tres grupos en estudio, los resultados fueron:, $858 \mathrm{hpg}$ para el grupo I (control), 891 hpg para el grupo II y de 875 en el grupo III. Luego del tratamiento, el grupo II tratado con IVM, presentó una disminución en el recuento de huevos de 483 hpg el día 7 y de 183 hpg en el día 15 diferencias que son estadísticamente significativa $(\mathrm{P}<0,05)$ con respecto al tiempo 0 (cuadro 1$)$. El grupo III, tratado con FBZ, disminuyó significativamente $(\mathrm{P}<0,05)$ sus promedios de recuento de huevos de nematodos a 433 hpg el día 7, y 209 hpg al día 15 (cuadro 1).

\section{PORCENTAJE DE EFICACIA ANTIHELMÍNTICA EN NE-} MÁTODOS GASTROINTESTINALES CON IVM Y FBZ

En el cuadro 2 se presentan los porcentajes de eficacia obtenidos posterior al tratamiento con IVM o FBZ. En el grupo de animales tratados con IVM se obtuvo un

Cuadro 1. Promedio de huevos de nematodos gastrointestinales por gramo de heces en borregas de raza Suffolk Down antes y después del tratamiento con IVM vía subcutánea o FBZ vía oral.

Mean values of faecal egg counts of gastrointestinal nematodes in Suffolk Down sheep-breed before and after treatment with IVM $(0.2 \mathrm{mg} /$ $\mathrm{kg}$ subcutaneously) or FBZ (5mg/kg, oral).

\begin{tabular}{cccc}
\hline $\begin{array}{c}\text { Tiempo } \\
\text { (Días) }\end{array}$ & $\begin{array}{c}\text { Grupo Control } \\
\text { (hpg) }\end{array}$ & $\begin{array}{c}\text { Grupo Ivermectina } \\
\text { (hpg) }\end{array}$ & $\begin{array}{c}\text { Grupo Fenbendazol } \\
(\mathrm{hpg})\end{array}$ \\
\hline 0 & $858 \pm 375,3$ (a) & $875 \pm 572,3$ (a) & $895 \pm 460,2(\mathrm{a})$ \\
7 & $733 \pm 461,9$ (a) & $483 \pm 212,5$ (b) & $433 \pm 210,3(\mathrm{~b})$ \\
15 & $808 \pm 1003,1$ (a) & $183 \pm 203,8$ (c) & $209 \pm 131,1(\mathrm{c})$ \\
\hline
\end{tabular}

\pm : Desviación estándar.

Letras diferentes en sentido vertical u horizontal indican diferencias significativas.

Cuadro 2. Porcentajes de eficacia a los días 7 y 15 post tratamiento, y límite inferior del intervalo de confianza al 95\%, para los grupos tratados con IVM y FBZ.

Percentage of efficacy and lower limit of 95\% confidence interval in the groups of sheep treated with IVM or FBZ.

\begin{tabular}{ccccc}
\hline \multirow{2}{*}{ Tiempo } & \multicolumn{2}{c}{ Grupo II, Ivermectina } & \multicolumn{2}{c}{ Grupo III, Fenbendazol } \\
\cline { 2 - 5 } & \% Eficacia & $* \mathrm{~L}_{\text {inf }}-\mathrm{IC}_{95 \%}$ & \% Eficacia & $* \mathrm{~L}_{\text {inf }}-\mathrm{IC}_{95 \%}$ \\
\hline Día 7 & 34 & 38 & 41 & 40 \\
Día 15 & 77 & & 74 & 40 \\
\hline
\end{tabular}

* $\mathrm{L}_{\mathrm{inf}}-\mathrm{IC}_{95 \%}$, Límite inferior del intervalo de confianza al 95\%, representa el valor de eficacia antihelmíntica para ambos periodos de muestreo. 
porcentaje de eficacia de $34 \%$ el día 7 , que aumenta a $77 \%$ el día 15. También en el grupo tratado con FBZ se obtuvieron porcentajes similares de $41 \%$ y $74 \%$, para los días 7 y 15, respectivamente.

El análisis estadístico para el cálculo de resistencia antihelmíntica, determinó que el $\mathrm{L}_{\text {inf }}-\mathrm{IC}_{95 \%}$ calculado para el porcentaje de eficacia en el grupo tratado con IVM fue de $38 \%$, mientras que en el grupo tratado con FBZ fue de $40 \%$ (cuadro 2).

Mediante la técnica de migración larvaria de Baermman realizada a los cultivos de muestras fecales para los grupos control y tratados con IVM o FBZ se obtuvieron un total de 97 larvas infectantes de tercer estado de nematodos gastrointestinales. De las cuales 72 pertenecieron al grupo control, entre ellas 28 del género Ostertagia y 44 a Trichostrongylus.

En los cultivos realizados para el grupo de animales tratados con IVM, los huevos no eclosionaron y no se recuperaron larvas L3.

De las larvas recuperadas de los cultivos del grupo de animales tratados con FBZ, se encontraron un total de 25 larvas de las cuales, 21 de ellas pertenecen al género Teladorsagia y 4 al género Trichostrongylus.

\section{DISCUSIÓN}

En el presente estudio se evaluó la eficacia antihelmíntica de los fármacos IVM y FBZ, como tratamiento para el control de nematodos gastrointestinales en borregas de 8 meses de edad. Los animales estudiados presentaron un recuento inicial promedio de $882 \mathrm{hpg}$, considerado como un nivel de parasitismo de tipo moderado (Barriga 2002). Este valor es superior a $150 \mathrm{hpg}$, que corresponde al mínimo establecido para realizar estudios de eficacia y resistencia a los antihelmínticos en ovinos (Coles y col 2006).

El porcentaje de reducción de huevos de nematodos obtenidos para el grupo IVM, alcanzó un máximo de $77 \%$ a los 15 días post tratamiento, porcentaje que es menor al $100 \%$ de eficacia descrito en corderos por Quitral en el año 2006. A su vez Pérez (2009), describe un 91\% de eficacia al día 15 de tratamiento en ovinos. En México, Montalvo y col (2006), describen porcentajes de reducción de $89,9 \%$ en la eliminación de huevos de nematodos gastrointestinales en ovejas. Por su parte, una pérdida total de eficacia describen González-Garduño y $\mathrm{col}^{2}$ en ovinos tratados con IVM en el estado de Tabasco, México. Por otra parte, valores similares a las del presente estudio, describen Suarez y Cristel (2007) en Argentina. con un $71,0 \%$ de eficacia de IVM sobre parásitos de bovinos.

\footnotetext{
González-Garduño R, G Torres-Hernández, MGJ Nuncio-Ochoa, JA Cuéllar-Ordaz, ME Zermeño-García 2003. Detección de eficiencia antihelmíntica en nemátodos de ovinos de pelo con la prueba de reducción de huevos en heces. Livestock Res. Rural Dev. 15 (12) http://www.lrrd.org/lrrd15/12/gonza1512.htm.
}

Según McKenna (2006), el obtener porcentajes de reducción de la ovoposición menores al 95\%, indicarían una sospecha de resistencia al fármaco o bien que la dosis $0,2 \mathrm{mg} / \mathrm{kg}$, sería insuficiente para alcanzar una buena respuesta antihelmíntica. De acuerdo a los criterios establecidos por la WAAVP, el segundo requisito que se debe cumplir para que exista resistencia, es que el límite inferior del intervalo al 95\% de confianza para el PRCH, sea menor de $90 \%$ (Coles y col., 2006). Por lo tanto, los resultados obtenidos en este estudio, demuestran que existe resistencia a IVM al presentar un porcentaje de eficacia menor al $95 \%$ y el límite inferior del intervalo de confianza del $95 \%$ menor al 90\%. La resistencia antihelmíntica a IVM se ha descrito en Argentina, Brazil, Uruguay y Paraguay (Nari, y col 1996), así como también en otros países del mundo (Borgsteede y col 2003).

Estudios realizados por Sievers y Alocilla, (2007). en bovinos del sur de Chile, han demostrado que en esta especie existe una baja efectividad de la IVM en el control de nemátodos gastrointestinales. Los principales generos de nematodos resistentes a este antihelmíntico fueron los Trichostrongylus, Cooperia y Nematodirus (Sievers y Alocilla 2007).

En los coprocultivos de muestras del grupo tratado con IVM, para la eclosión y posterior recuperación de larvas de nemátodos, no se observó desarrollo larvario, por lo que no fue posible caracterizar los principales géneros parasitarios que son resistentes a este antihelmíntico. Al respecto, la literatura describe que los géneros parasitarios que presentan una mayor frecuencia de resistencia antihelmíntica a las avermectinas son Teladorsagia y Haemonchus (Bartley y col 2006).

En las borregas de este estudio tratadas con FBZ, se obtuvieron porcentajes de reducción del recuento de huevos de nemátodos, de $74 \%$. Estos resultados contrastan con los descritos en el primer estudio realizado en Chile, sobre el uso de FBZ en ovinos Suffolk Down de la provincia de Bío Bío, realizado por Becker y col (1979), en el que se encontró una eficacia sobre nematodos gastrointestinales igual o superior al 99,2\%. Otro estudio efectuado en ovinos de 8 meses de edad y tratados con FBZ (Gonzáles 1980) demostró reducción del 100\%, a los 15 días después del tratamiento. Similares resultados describe Hovorka y col (1975), al administrar FBZ en dosis de $5 \mathrm{mg} / \mathrm{kg}$ de peso en cabras, en las que se alcanzó un $100 \%$ de efectividad en la eliminación de nemátodos gastrointestinales al día 7 pos tratamiento.

Similar al grupo tratado con IVM, las borregas del grupo tratado con FBZ presentaron porcentaje de reducción de $74 \%$, valor que es menor al $95 \%$ recomendado por la WAAVP para establecer eficacia. Además, el límite inferior del intervalo al $95 \%$ de confianza fue de $40 \%$. Por lo tanto, en el grupo de borregas tratadas con este antihelmíntico se considera que existe resistencia a la dosis oral de $5 \mathrm{mg} / \mathrm{kg}$ de FBZ.

Las larvas recuperadas del cultivo de heces muestran que para el grupo tratado con FBZ se recuperaron 25 lar- 
vas infestantes de las cuales 21 correspondieron al género Ostertagia y 4 a Trichostrongylus.

Estudios en ovinos tratados con benzimidazoles, reportan resistencia de nematodos gastrointestinales, en los géneros Trichostrongylus spp, Ostertagia spp y Haemonchus (Kaplan, 2004, Wolstenholme y col 2004), y Oesophagostomum (Márquez 2003).

La comparación de los resultados del presente estudio con aquellos reportados en la literatura muestran que con el transcurso de los años, en el país se ha producido una disminución de la eficacia de estos antihelmínticos sobre los parásitos gastrointestinales de los ovinos. Trabajos realizados por Quitral en al año 2006, demostraron $100 \%$ de eficacia de IVM sobre nematodos gastrointestinales de ovinos. Luego en el año 2009 se describe una eficacia de $91 \%$ (Pérez 2009), continuando la reducción de eficacia en el presente estudio donde se alcanzó un $77 \%$. Lo mismo se observa para FBZ, que en el año 1980 presentaba porcentajes de $100 \%$ de eficacia (Becker y col 1979, González 1980), hasta observar actualmente en el presente estudio una eficacia de $74 \%$. Estos antecedentes sugieren que con el transcurso de los años y debido probablemente al uso indiscriminado se ha producido una pérdida paulatina de eficacia tanto de IVM como de FBZ, frente a los nematodos gastrointestinales de los ovinos. Bajo estas circunstancias, se concluye que existe una clara necesidad de implementar programas de monitoreo para evaluar la eficacia de los antihelmínticos como requisito esencial para desarrollar programas de control parasitario en la especie ovina que sean efectivos.

En el país no existen antecedentes previos que describan la presencia de resistencia parasitaria en ovinos a los efectos antihelmínticos de IVM y FBZ, por lo cual se requiere realizar nuevos estudios que incluyan un mayor número de animales con el fin de conocer el estado sanitario de la región y del país e informar a los agricultores, médicos veterinarios y personas relacionadas con la actividad ganadera, acerca de la importancia de la correcta utilización de los antihelmínticos así como de las consecuencias del uso indiscriminado de estos fármacos.

\section{REFERENCIAS}

Barriga O. 2002. Las enfermedades parasitarias de los animales domésticos en la América latina. Editorial Germinal, Santiago, Chile.

Bartley DJ, AA Donnan, E Jackson, N Sargison, GBB Mitchell, F Jackson. 2006. A small scale survey of ivermectin resistance in sheep nematodes using the faecal egg count reduction test on samples collected from Scottish sheep. Vet Parasitol 137, 112-118.

Becker F, H Alcaíno, T Gorman. 1979. Evaluación del poder antihelmintico de fenbendazole, tetramizole y levamisole contra nematodos gastronitestinales de ovinos. Arch Med Vet Supplem. 1, 129-133.

Borgsteede FHM, DD Dercksen, R Huibers. 2003. Doramectin and albendazole resistance in sheep in the Netherlands.
Vet Parasitol 144, 180-183.

Coles G, C Bauer, F Borgsteede, S Geerts, T Klei, M Taylor, P Waller. 1992. World Association for the Advancement of Veterinary Parasitology (WAAVP) methods for the detection of anthelmintic resistance in nematodes of veterinary importance. Vet. Parasitol 44, 35-44.

Coles GC, FW Jackson, E Pomroy, RK Prichard, G von Samson-Himmelstjerna, A Silvestre, MA Taylor. 2006. The detection of anthelmintic resistance in nematodes of veterinary importance. Vet Parasitol 136, 167-185.

Cristel S, V Suarez. 2006. Resistencia antihelmíntica, evaluación de la prueba de reducción del conteo de huevos. RIA $35,29-43$.

Donald AD. 1974. Some recent advances in the epidemiology and control of helminth infection in sheep. Proc Aust Soc Anim Prod 10, 148-175.

FAO, Food and Agriculture Organization of the United Nations. 2003. Resistencia a los antiparasitarios. Estado actual con énfasis en América Latina. Estudio FAO Producción y Sanidad Animal 157, 1-51.

González H. 1980. Actividades de antihelminticos contra parasitos gastrointestinales en ovinos. Monografías de Medicina Veterinaria 2, 7-26.

Hovorka J, J Mitterpák, J Corba, R. Spaldonová, H Pacenovský. 1975. Effect of fenbendazole (Panacur) in sheep naturally invaded by gastrointestinal and lung nemtaodes. Vet Med (Praha) 20, 391-7.

Jackson F, R Coop. 2000. The development of anthelmintic resistance in sheep nematodes. Parasitol 120, 95-107.

Kaplan R. 2004. Drug resistance in nematodes of veterinary importance: a status report. Trends Parasitol 20, 477-481.

Márquez D. 2003. Resistencia a los antihelmínticos, origen, desarrollo y control. Corpoica 4, 55-71.

McKenna P. 2006. A comparison of faecal egg count reduction test procedures. $N$ Z Vet J 54, 202-203.

Montalvo, X, M López, V Vásquez, E Liébano, P Mendoza. 2006. Resistencia antihelmíntica de nematodos gastroentéricos en ovinos a fenbendazol e ivermectina en la región noroeste del estado de Tlaxcala. Те́c Реси Мéx 44, 81-90.

Nari A, J Salles, A Gil, P Waller, J Hansen. 1996. The Prevalence of anthelmintic resistance in nematode parasites of sheep in Southern Latin America, Uruguay. Vet Parasitol 62, 213-222.

Niec R. 1968. Cultivo e identificación de larvas infectantes de nematodes gastrointestinales del bovino y ovino. Manual técnico $N^{\circ}$ 3. INTA, Argentina, Pp 1-37.

Pérez RA. 2009. Evaluación de eficacia de ivermectina asociada con vitaminas A, D3, E (INVECTINA ADE®) en el tratamiento de parásitos gastrointestinales y la ganancia de peso en corderos naturalmente infectados. Memoria de Titulo, Escuela de Medicina Veterinaria, Universidad de Concepción, Concepción, Chile.

Pérez R. 2011. Bases farmacológicas de la terapéutica antihelmíntica en rumiantes: Eficacia y seguridad de avermectinas y milbemicinas. Anales del XII Congreso Chileno de Buiatria, Osorno, Chile, Pp 49-58.

Quitral B. 2006. Comparación de la eficacia antihelmíntica entre ivermectina y doramectina contra parasitismo gastrointestinal en ovinos. Memoria de Titulo, Escuela de Medicina Veterinaria, Universidad de Concepción, Con- 
cepción, Chile.

Sievers G, C Fuentealba. 2003. Comparación de la efectividad antihelmíntica de seis productos comerciales que contienen lactonas macrocíclicas frente a nematodos gastrointestinales del bovino. Arch Med Vet 35, 81-88.

Sievers G, A Alocilla. 2007. Determinación de resistencia antihelmíntica frente a ivermectina de nematodos del bovino en dos predios del sur de Chile. Arch Med Vet 35, 81-88.

Suarez VH, SL Cristel. 2007. Anthelmintic resistance in cattle nematode in the western Pampeana Region of Argentina. Vet Parasitol 144, 11-117.

Van Wyk JA. 2001. Refugia-overlooked as perhaps the most potent factor concerning the development of anthelmintic resistance. Onderstepoort J Vet Res 68, 55-67.

Williams J. 1997. Anthelmintic treatment strategies: current status and future. Vet Parasitol 72, 461-477.

Witzendorff von C, I Quintana, G Sievers, T Schnieder y G von Samson-Himmelstjerna. 2003. Estudio sobre resistencia frente a los bencimidazoles de pequeños estróngilos (Cyathostominae) del equino en el sur de Chile. Arch Med Vet 35, 187-194.

Wolstenholme A, I Fairweather, R Prichard, G Samson, N Sangster. 2004. Drug resistance in veterinary helminths. Trends Parasitol 20, 469-476. 